\title{
Two Convergent Triangle Tunnels
}

\section{Two Convergent Triangle Tunnels \\ ABSTRACT}

A semi-orthogonal path is a polygon inscribed into a given polygon such that the $i$-th side of the path is orthogonal to the $i$-th side of the given polygon. Especially in the case of triangles, the closed semi-orthogonal paths are triangles which turn out to be similar to the given triangle. The iteration of the construction of semi-orthogonal paths in triangles yields infinite sequences of nested and similar triangles. We show that these two different sequences converge towards the bicentric pair of the triangle's Brocard points. Furthermore, the relation to discrete logarithmic spirals allows us to give a very simple, elementary, and new constructions of the sequences' limits, the Brocard points. We also add some remarks on semi-orthogonal paths in non-Euclidean geometries and in $n$-gons.

Key words: triangle, semi-orthogonal path, Brocard points, symmedian point, discrete logarithmic spiral, Tucker-Brocard cubic

MSC2010: 51A05, 51A20

\section{Introduction}

\subsection{Sequences of triangles}

Little is known about sequences of Cevian triangles within a given triangle. Sequences of medial triangles and Routh triangles are studied in [3]. There, triangles are considered as triplets of points in the complex plane and a shape function which is actually a complex affine ratio is defined and describes how the shape of a triangle changes during the iteration process. It turns out that the above mentioned classes of triangles converge in shape, in most cases to equilateral triangles.

It is well-known (and rather trivial) that the sequence of Cevian triangles of a triangle's centroid converges towards the centroid. The intouch triangle (contact points of the in-

\section{Dva konvergentna niza trokuta}

\section{SAŽETAK}

Poluortogonalan put je poligonalna linija upisana u dani mnogokut takva da je $i$-ta stranica poligonalne linije okomita na $i$-tu stranicu danog mnogokuta. U slučaju trokuta, zatvoreni poluortogonalni putovi su trokuti slični danom trokutu. Iteracijom konstrukcije poluortogonalnih putova u trokutima dobivaju se beskonačni nizovi upisanih sličnih trokuta. Pokazujemo da ova dva različita niza konvergiraju prema bicentričnom paru Brocardovih točaka trokuta. Nadalje, veza s diskretnim logaritamskim spiralama omogućuje vrlo jednostavnu, elementarnu i novu konstrukciju limesa ovih nizova, Brocardovih točaka. Iznosimo i neke napomene o poluortogonalnim putovima kako u neeuklidskim geometrijama i tako i za $n$-kute.

Ključne riječi: trokut, poluortogonalan put, Brocardove točke, sjecište simedijana, diskretna logaritamska spirala, Tucker-Brocardova kubika

circle and the triangle sides) is always in the interior of the initial triangle (cf. Fig. 1). So, it is nearby to expect that the sequence of nested intouch triangles has a point shaped limit which is yet undiscovered. On the contrary, the orthic triangle is an interior triangle only if the base triangle is acute and, unfortunately, acute triangles may have an obtuse orthic triangle and convergence cannot be expected in the generic case.

We try to leave the beaten tracks by starting the construction of the triangles of the sequence in a different way. The edges of the triangles in the sequences in question shall form a semi-orthogonal path, i.e., the $i$-th edge of the new triangle shall be orthogonal to the $i$-th side of the given triangle. Depending on the ordering of the sides of the base triangle, we find two closed semi-orthogonal paths which 
shall be constructed and discussed in Sec. 2. Further, the case of generic $n$-laterals ( $n$ straight lines in generic position such that no two lines enclose a right angle) shall be addressed in Sec. 2, besides some comments on closed semi-orthogonal paths in non-degenerate Cayley-Klein geometries, i.e., the elliptic and the hyperbolic plane. Sec. 3 is dedicated to the computation of the limits of the triangle sequences. We show that the triangles in one sequence shrink to one Brocard point, while the others converge to the other Brocard point, and thus, these two limits are located on the Tucker-Brocard cubic. Finally, in Sec. 4, we conclude and address some open problems. The remaining part of this section (Sec. 1) collects some prerequisites.

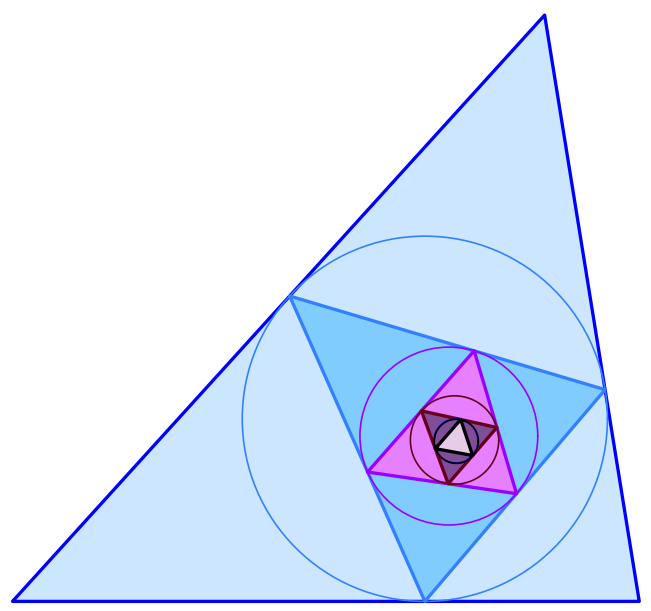

Figure 1: Where and what is the limit of the sequence of intouch triangles?

\subsection{Prerequisites and conventions}

Since we deal with triangles in the Euclidean plane $\mathbb{R}^{2}$, we use Cartesian coordinates in order to describe points. It will turn out useful to perform the projective closure of the Euclidean plane by adding the ideal line $\omega$ to $\mathbb{R}^{2}$. Whenever, we deal with points and lines in the projectively extended plane, we can switch between Cartesian and homogeneous coordinates of points by

$$
(1, x, y) \longleftrightarrow\left(x_{0}: x_{1}: x_{2}\right)
$$

as long as $x_{0} \neq 0$. Lines $l: a_{0}+a_{1} x+a_{2} y=0$ can also be described by homogeneous coordinates $\left(a_{0}: a_{1}: a_{2}\right)$. Especially, the ideal line (or line at infinity) is simply given by $\omega=(1: 0: 0)$.

For the moment, it is sufficient to assume that the Cartesian coordinates of the vertices of the base triangle $\Delta_{0}$ are

$A_{0}=(0,0), B_{0}=(c, 0), C_{0}=(u, v)$.
We assume that $c, v \neq 0$ so that $A_{0} \neq B_{0}$ and $C_{0} \notin\left[A_{0}, B_{0}\right]$. Further, $u^{2}+v^{2} \neq 0$ which implies $A_{0} \neq C_{0}$. In the following, no interior angle of $\Delta_{0}$ shall be a right one. This is expressed algebraically by $u \neq c, u \neq 0$, and $u^{2}-u c+v^{2} \neq 0$.

We shall agree that the side lengths of $\Delta_{0}$ are

$$
a:=\overline{B_{0} C_{0}}, \quad b:=\overline{C_{0} A_{0}}, c:=\overline{A_{0} B_{0}} .
$$

Later, when we try to express especially metric properties of the triangle in terms of $\Delta_{0}$ 's side lengths $a, b, c$, we should be able to replace $u$ and $v$ from (1) by functions depending on $a, b, c$. For that purpose, we compute $C_{0}=(u, v)$ as the intersection of two circles: one centered at $A_{0}$ with radius $b$; the other one centered at $B_{0}$ with radius $a$ such that $v>0$. This results in

$u=\frac{b^{2}+c^{2}-a^{2}}{2 c}$ and $v=\frac{2 F}{c}$

with $F$ being the area of $\Delta_{0}$. Note that $F$ can be expressed in terms of $\Delta_{0}$ 's side lengths using Heron's formula or, equivalently, with help of the Cayley-Menger determinant.

\section{Closed semi-orthogonal paths}

\subsection{Triangles in the Euclidean plane}

Let $\Delta_{0}=A_{0} B_{0} C_{0}$ be a triangle in the Euclidean plane. Let further $P_{0}$ be a point on the side line $\left[A_{0}, B_{0}\right]$. We construct a sequence $P_{0}, P_{1}, P_{2}, P_{3}$ of points on the lines $\left[A_{0}, B_{0}\right]$, $\left[B_{0}, C_{0}\right],\left[C_{0}, A_{0}\right],\left[A_{0}, B_{0}\right]$ in the following way (cf. Fig. 2):

$$
\begin{aligned}
& P_{0} \in\left[A_{0}, B_{0}\right],\left[P_{0}, P_{1}\right] \perp\left[A_{0}, B_{0}\right], P_{1} \in\left[B_{0}, C_{0}\right] \\
& \text { with } P_{0} \rightarrow P_{1} \rightarrow P_{2} \rightarrow P_{3}, A_{0} \rightarrow B_{0} \rightarrow C_{0} \rightarrow A_{0},
\end{aligned}
$$

cyclical replacement.

Henceforth, we shall refer to such paths as semiorthogonal paths. It doesn't make a difference if we start at $\left[A_{0}, B_{0}\right]$ or at any other side line of $\Delta_{0}$.

Obviously, the mapping $\pi: P_{0} \mapsto P_{3}$ is a projective mapping $\left[A_{0}, B_{0}\right] \rightarrow\left[A_{0}, B_{0}\right]$, since it is a chain of three perspectivities:

$$
\underbrace{\left[A_{0}, B_{0}\right] \frac{R^{\perp}}{\pi}\left[B_{0}, C_{0}\right] \frac{S^{\perp}}{\pi}\left[C_{0}, A_{0}\right] \frac{T^{\perp}}{\pi}\left[A_{0}, B_{0}\right]}_{\pi}
$$

where $R:=\left[A_{0}, B_{0}\right] \cap \omega, S:=\left[B_{0}, C_{0}\right] \cap \omega$, and $T:=$ $\left[C_{0}, A_{0}\right] \cap \omega$ are the ideal points of $\Delta_{0}$ 's side lines and $R^{\perp}$, $S^{\perp}$, and $T^{\perp}$ are the ideal points of the respective orthogonal directions. Note, that the three perspectors are collinear as indicated in Fig. 2. 


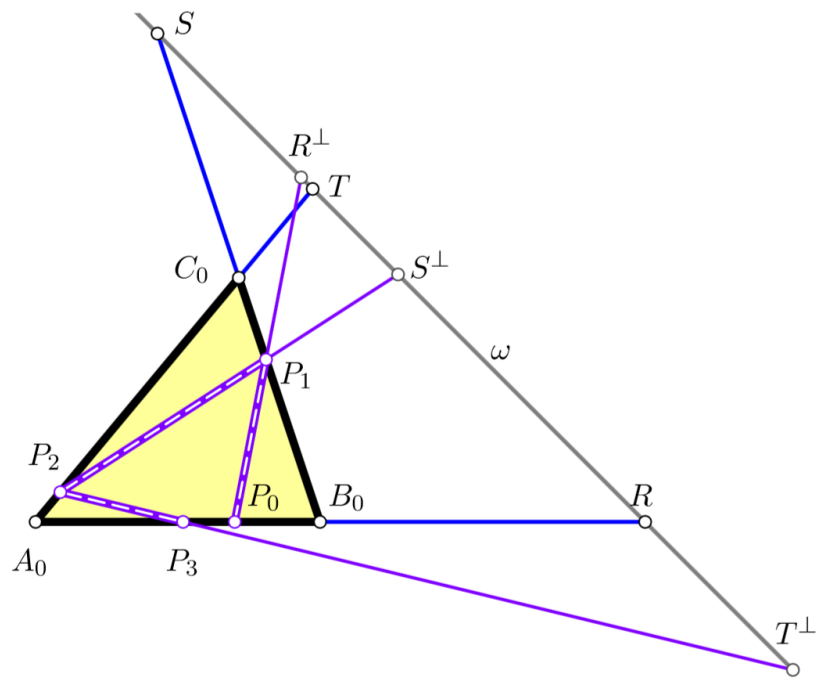

Figure 2: The projective mapping $\pi:\left[A_{0}, B_{0}\right] \rightarrow\left[A_{0}, B_{0}\right]$ is the product of three perspectivities. The perspectors themselves are assigned to the ideal points of $\Delta_{0}$ 's side lines in a projective way: They are joined by the absolute polarity acting on $\omega$.

Remark 1 Indeed, the projective mapping $\perp: \omega \rightarrow \omega$ that assigns the ideal point of the orthogonal direction to any ideal point can be replaced by any other elliptic projective mapping acting on $\omega$. The pseudo-Euclidean case would be covered if $\perp$ is hyperbolic.

The ideal point $R$ of $\left[A_{0}, B_{0}\right]$ is self-assigned in $\pi$, since $\pi(R)=R$, and therefore, we can expect only one further fixed point $A_{1}$ of $\pi$.

Let now, $P_{0}=(t, 0)$ with $t \in \mathbb{R}$ which is a parametrization of the line $\left[A_{0}, B_{0}\right]$ and we find

$$
\begin{gathered}
P_{1}=\left(t, \frac{v(c-t)}{c-u}\right), P_{2}=\frac{\alpha(t)}{\beta}(u, v), \\
P_{3}=\frac{\alpha(t)\left(u^{2}+v^{2}\right)}{u \beta}(1,0)
\end{gathered}
$$

where

$$
\begin{aligned}
\alpha(t) & =\left((c-u)^{2}+v^{2}\right) t-c v^{2}, \\
\beta & =\left((c-u)^{2}+v^{2}\right) u-c v^{2} .
\end{aligned}
$$

Note that $c-u$ is a divisor of $\beta$ that cannot vanish due to assumptions made earlier. The path $P_{0} P_{1} P_{2} P_{3}$ is closed if the points $P_{0}$ and $P_{3}$ coincide. This is equivalent to $t=\frac{\alpha(t)}{u \beta}\left(u^{2}+v^{2}\right)$, and thus,

$t=\frac{c\left(u^{2}+v^{2}\right)}{c^{2}-c u+u^{2}+v^{2}}$.
We shall make explicit the fact that the denominator of $t$ in (4) cannot vanish: Substituting (2) into (4), we find

$t=2 b^{2} c \sigma^{-1}$

where

$\sigma:=a^{2}+b^{2}+c^{2}$

which cannot vanish for $a, b, c \in \mathbb{R}^{\star}$.

If we insert (4) into (3) and relabel the points by letting $A_{1}=P_{0}=P_{3}, B_{1}=P_{1}$, and $C_{1}=P_{2}$, we arrive at

$$
\begin{gathered}
A_{1}=\frac{c}{\gamma}\left(c\left(u^{2}+v^{2}\right), 0\right), \\
B_{1}=\frac{c}{\gamma}\left(u^{2}+v^{2}, c v\right), C_{1}=\frac{c u}{\gamma}(u, v)
\end{gathered}
$$

with $\gamma=c^{2}-c u+u^{2}+v^{2}$. Such a closed triangular path $A_{1} B_{1} C_{1}$ is shown in Fig. 3 .

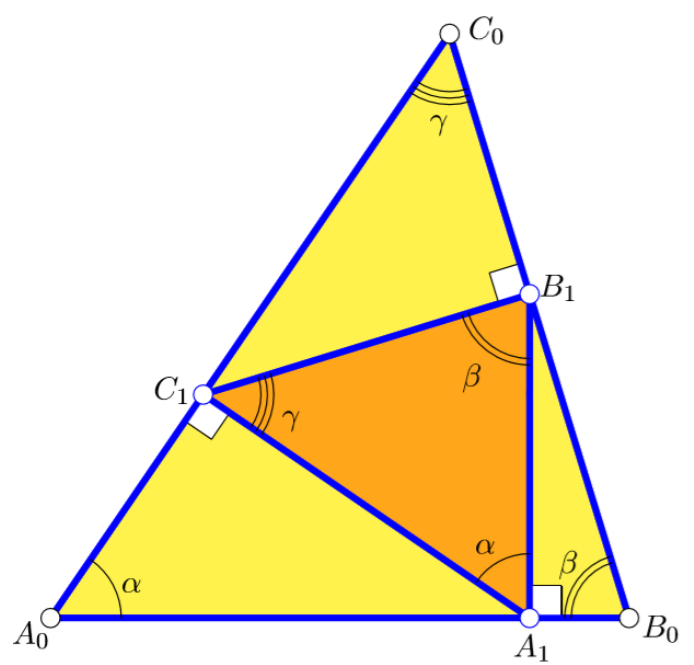

Figure 3: The first triangle $\Delta_{1}=A_{1} B_{1} C_{1}$ inscribed into $\Delta_{0}=A_{0} B_{0} C_{0}$.

As can be seen in Fig. 3,

$$
\begin{aligned}
& \Varangle C_{1} A_{1} B_{1}=\Varangle C_{0} A_{0} B_{0}, \\
& \Varangle A_{1} B_{1} C_{1}=\Varangle A_{0} B_{0} C_{0}, \\
& \Varangle B_{1} C_{1} A_{1}=\Varangle B_{0} C_{0} A_{0} .
\end{aligned}
$$

Thus, we have

Lemma 1 The triangles $\Delta_{0}$ and $\Delta_{1}$ are similar.

In order to construct the path $A_{1} B_{1} C_{1}$, we started at $P_{0}$ leaving $\left[A_{0}, B_{0}\right]$ in the orthogonal direction until we meet $\left[B_{0}, C_{0}\right]$. We could also look for a path $Q_{0} Q_{1} Q_{2} Q_{3}$ with 
$Q_{0}=P_{0}$ and $Q_{1} \in\left[C_{0}, A_{0}\right]$, i.e., leaving $\left[A_{0} B_{0}\right]$ in the orthogonal direction until we meet $\left[C_{0} A_{0}\right]$, and so forth. This yields a second closed triangular path if we achieve $Q_{0}=Q_{3}$. Then, $L_{1}=Q_{0}, K_{1}=Q_{1}$, and $M_{1}=Q_{2}$ form a triangle $\nabla_{1}$, see Fig. 4. Similar to Lem. 1 and due to the same reasoning, we have

Lemma 2 The triangles $\Delta_{0}$ and $\nabla_{1}$ are similar.

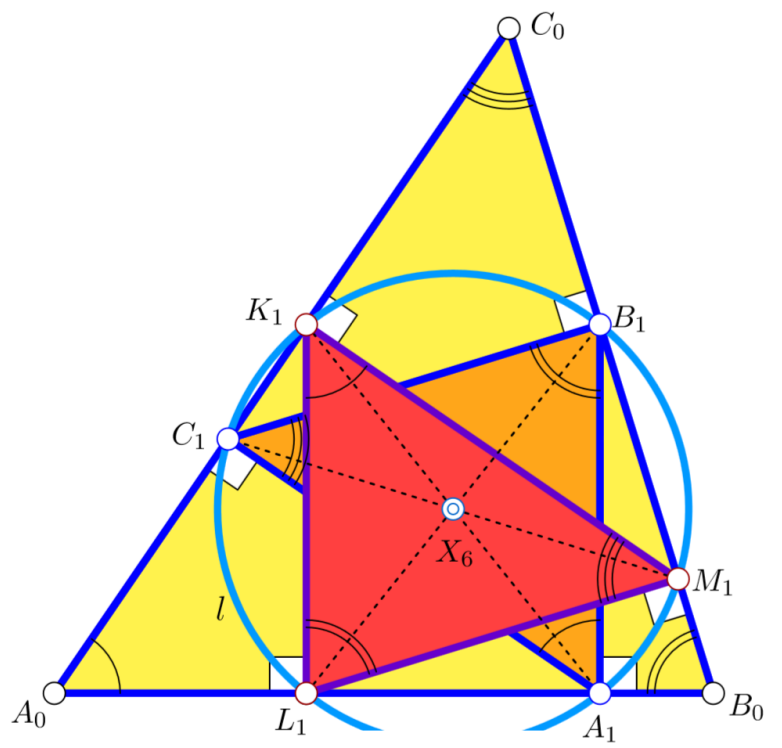

Figure 4: The two perspective triangles $\Delta_{1}$ and $\nabla_{1}$ with their common circumcircle (centered at the symmedian point $\left.X_{6}\right)$.

Moreover, we can show the following

Theorem 1 The triangles $\Delta_{1}$ and $\nabla_{1}$ are congruent, share the circumcircle $l$, and therefore, the circumcenter which is the symmedian point $X_{6}$ of $\Delta_{0}{ }^{1}$

Proof. The similarity of $\Delta_{0}$ and $\Delta_{1}$ needs no further confirmation, since this is done right before Lem. 1. The similarity of $\Delta_{0}$ and $\nabla_{1}$ can be shown in the same way. From $\Delta_{1} \sim \Delta_{0}$ and $\nabla_{1} \sim \Delta_{0}$ we can infer $\Delta_{1} \sim \nabla_{1}$.

Since $C_{1} M_{1}$ is seen from $B_{1}$ and $K_{1}$ at right angles, $B_{1}, C_{1}$, $K_{1}$, and $M_{1}$ are concyclic. Further, $B_{1} L_{1}$ is seen from $A_{1}$ and $M_{1}$ at right angles. Thus, the circumcircle of $B_{1}, C_{1}$, $K_{1}$, and $M_{1}$ equals that of $A_{1}, B_{1}, L_{1}$, and $M_{1}$.

Two similar triangles can only share the circumcircle if they are congruent (which can be confirmed with help of the Law of sines).

Finally, we have to show that the circumcenter of the six points $A_{1}, B_{1}, C_{1}, K_{1}, L_{1}, M_{1}$ is the symmedian point $X_{6}$ of
$\Delta_{0}$. We compute the actual distances of the midpoint $M$ of the segment $A_{1} K_{1}$ to $\Delta_{0}$ 's side lines and find

$$
\begin{aligned}
& \overline{M\left[A_{0}, B_{0}\right]}=2 c F \sigma^{-1}, \\
& \overline{M\left[B_{0}, C_{0}\right]}=2 a F \sigma^{-1}, \\
& \overline{M\left[C_{0}, A_{0}\right]}=2 b F \sigma^{-1},
\end{aligned}
$$

and thus, the homogeneous trilinear coordinates are

$$
M=(a: b: c)
$$

which confirms that $M$ equals the symmedian point $X_{6}$ of $\Delta_{0}$.

From Lem. 1, we can deduce a simple linear construction of $\Delta_{1}$ (and $\nabla_{1}$ ) which is shown in Fig. 5: An arbitrary triangle $\Delta=A^{\prime} B^{\prime} C^{\prime}$ with

$$
\begin{gathered}
A^{\prime} \in\left[A_{0}, B_{0}\right], B^{\prime} \in\left[B_{0}, C_{0}\right], \\
{\left[A^{\prime}, B^{\prime}\right] \perp\left[A_{0}, B_{0}\right]}
\end{gathered}
$$

similar to $\Delta_{0}$ is drawn, i.e.,

$$
\begin{aligned}
& \Varangle C^{\prime} A^{\prime} B^{\prime}=\Varangle C_{0} A_{0} B_{0}, \\
& \Varangle A^{\prime} B^{\prime} C^{\prime}=\Varangle A_{0} B_{0} C_{0} .
\end{aligned}
$$

The central similarity with center $B_{0}$ sends $C^{\prime}$ to $C_{1} \in$ $\left[C_{0}, A_{0}\right]$, and thus, it maps $\Delta^{\prime} \rightarrow \Delta_{1}$.

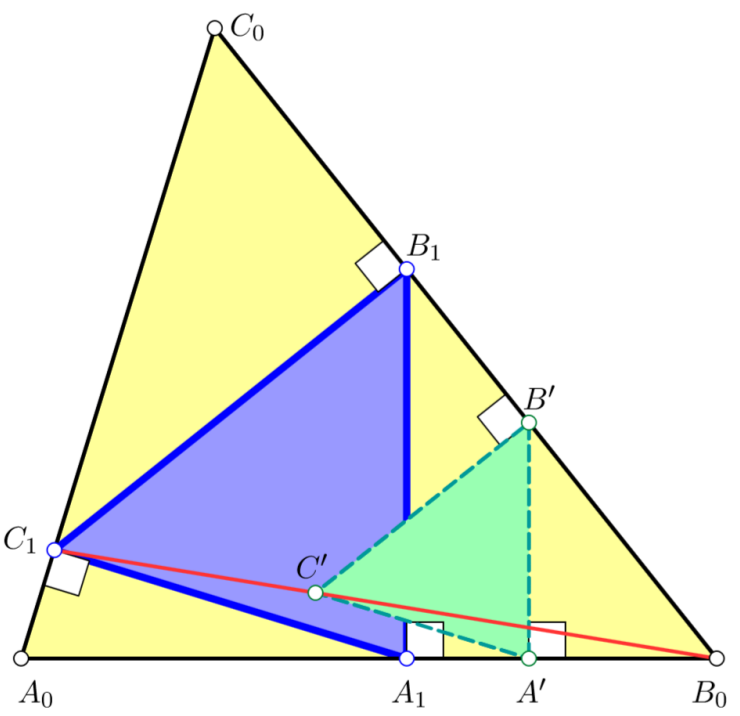

Figure 5: The construction of $\Delta_{1}$ is linear and uses the central similarity from $B_{0}$.

From this construction it is clear that there is a second triangle $\nabla_{1}=K_{1} L_{1} M_{1}$ similar to $\Delta_{0}$ and inscribed into $\Delta_{0}$,

\footnotetext{
${ }^{1}$ Here, and in the following $X_{i}$ means the $i$-th point in Kimberlings's encyclopedia of triangle centers [4, 5].
} 
but different from $\Delta_{1}$ : We start with a triangle $\nabla=K^{\prime} L^{\prime} M^{\prime}$ and let

$$
\begin{gathered}
L^{\prime} \in\left[A_{0}, B_{0}\right], K^{\prime} \in\left[C_{0}, A_{0}\right], \\
{\left[K^{\prime}, L^{\prime}\right] \perp\left[A_{0}, B_{0}\right]}
\end{gathered}
$$

with

$$
\begin{aligned}
& \Varangle K^{\prime} L^{\prime} M^{\prime}=\Varangle A_{0} B_{0} C_{0}, \\
& \Varangle M^{\prime} K^{\prime} L^{\prime}=\Varangle C_{0} A_{0} B_{0} .
\end{aligned}
$$

Now, the similarity with center $A_{0}$ sends $K^{\prime} L^{\prime} M^{\prime}$ to $K_{1} L_{1} M_{1}$.

\subsection{Non-Euclidean planes}

The case of the pseudo-Euclidean plane was the subject of Rem. 1 since its only difference is the hyperbolic projectivity on the ideal line (in the plane's projective extension).

In hyperbolic and elliptic geometry, there is still a projective mapping $\pi:\left[A_{0}, B_{0}\right] \rightarrow\left[A_{0}, B_{0}\right]$. However, since there is no ideal line, but rather an ideal conic, we miss a selfassigned ideal point on $\left[A_{0}, B_{0}\right]$. Thus, $\pi$ has up to two real fixed points:

Theorem 2 In each generic triangle in the hyperbolic or elliptic plane, there exist two closed semi-orthogonal paths for a particular chosen ordering of side lines.

The reality of the fixed points mentioned in Thm. 2 is clear in the hyperbolic and in the elliptic case: Let $\omega$ denote the absolute conic. Each pair $\left(V_{i}, V_{j}\right)$ of proper vertices of the triangle and the pair of absolute points $\left(A_{1}, A_{2}\right)=$ $\left[V_{i}, V_{j}\right] \cap \omega$ of $\left[V_{i}, V_{j}\right]$ are not entangled. Therefore, there are two real fixed points on $\left[V_{i}, V_{j}\right]$, see $[2$, p. 254].

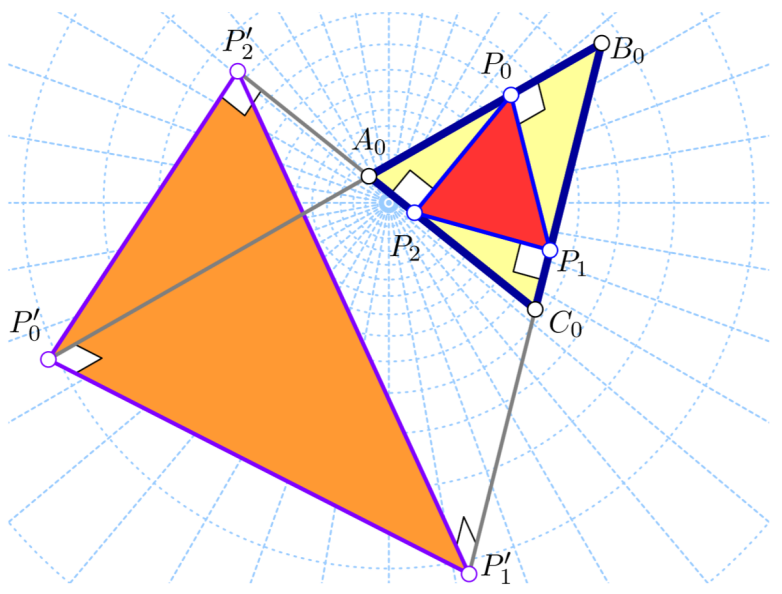

Figure 6: Two closed semi-orthogonal paths in a triangle $\Delta_{0}=A_{0} B_{0} C_{0}$ of the elliptic plane. The paths are starting with $\left[P_{0}, P_{1}\right] \perp_{\mathrm{e}}\left[A_{0}, B_{0}\right]$ and $\left[P_{0}^{\prime}, P_{1}^{\prime}\right] \perp_{\mathrm{e}}\left[A_{0}, B_{0}\right]$, respectively.
Fig. 6 shows a triangle $\Delta_{0}=A_{0} B_{0} C_{0}$ in the projective model of the elliptic plane together with the two closed semi-orthogonal paths to a chosen ordering of $\Delta_{0}$ 's side lines.

There are two different closed semi-orthogonal paths in a triangle in the elliptic or in the hyperbolic plane. Since the projective mapping $\pi:\left[A_{0}, B_{0}\right] \rightarrow\left[A_{0}, B_{0}\right]$ is hyperbolic in elliptic as well as hyperbolic geometry, there are four closed semi-orthogonal (triangular) paths in a generic triangle. Fig. 7 shows these four closed semi-orthogonal paths in a triangle in the elliptic plane. This four-fold symmetry resembles the four-fold symmetry in Universal Hyperbolic Geometry (cf. $[8,9]$ ) and shows up when classical triangle geometry is also studied from the viewpoint of projective geometry, see [7].

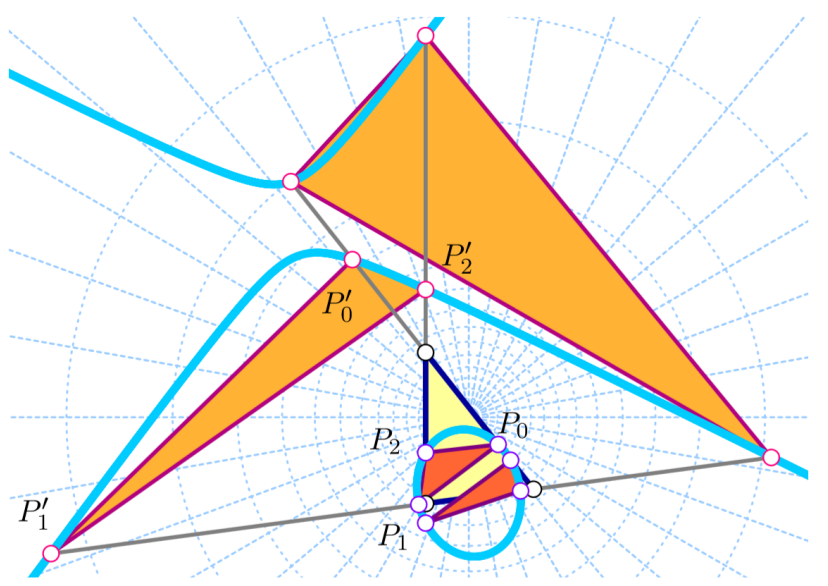

Figure 7: There are four closed semi-orthogonal paths in a triangle in the elliptic plane. Two by three vertices gather on a conic: three points from an $\left[A_{0}, B_{0}\right]-\left[B_{0}, C_{0}\right]-\left[C_{0}, A_{0}\right]$ path and three vertices from an $\left[A_{0}, B_{0}\right]-\left[C_{0}, A_{0}\right]-\left[B_{0}, C_{0}\right]$ path.

Neither in the elliptic nor in the hyperbolic plane we can use Thales's theorem in order to show that two of the triangular paths share a circumconic as illustrated in Fig. 7.

\section{Infinite sequences of inscribed triangles}

In this section, we return to Euclidean geometry in order to attack the main problem.

We have seen that $\Delta_{0}$ and $\Delta_{1}$ are similar triangles. Consequently, the triangle $\Delta_{2}$ inscribed into $\Delta_{1}$ whose vertices $A_{2}, B_{2}, C_{2}$ are obtained in the same way as $A_{1}, B_{1}, C_{1}$ is also similar to $\Delta_{1}$, and thus, to $\Delta_{0}$. This procedure can be repeated arbitrarily often which yields a sequence of similar triangles $\Delta_{0}, \Delta_{1}, \Delta_{2}, \Delta_{3}, \Delta_{4}, \ldots$ for a particular triangle $\Delta_{0}$. 
Due to the construction of $\Delta_{1}$, subsequent edges $A_{i} A_{i+1}$ and $A_{i+1} A_{i+2}$ of the polygon $A_{0} A_{1} A_{2} A_{3} A_{4} \ldots$ are orthogonal. Further, the edges $A_{i} A_{i+1}$ and $A_{i+2} A_{i+3}$ are antiparallel. The same holds true for the polygons $B_{0} B_{1} B_{2} \ldots$ and $C_{0} C_{1} C_{2} \ldots$.

Fig. 8 shows the polygon $A_{0} A_{1} A_{2} A_{3} \ldots$, while Fig. 9 shows the six discrete logarithmic spirals encircling two different limits.

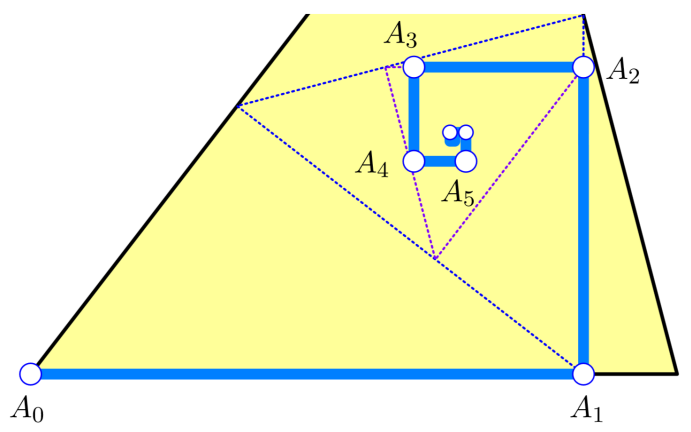

Figure 8: The discrete logarithmic spiral formed by $A_{0}$, $A_{1}, \ldots$ winding towards a limit.

Now, we want to show that the triangles $\Delta_{i}$ and $\nabla_{i}$ converge to a point as $i \rightarrow \infty$. In this case, it is not necessary to apply shape functions like in [3]. We need some other prerequisites. With (7) it is easy to verify that the side lengths $a_{1}=\overline{B_{1} C_{1}}, b_{1}=\overline{C_{1} A_{1}}, c_{1}=\overline{A_{1} B_{1}}$ of $\Delta_{1}$ are

$$
a_{1}=a \lambda, \quad b_{1}=b \lambda, \quad c_{1}=c \lambda
$$

where $\lambda$ is the scaling factor of the similarity $\Delta_{0} \rightarrow \Delta_{1}$ which can be computed from $a, b$, and $c$ via

$\lambda=4 F \sigma^{-1}$

where $F$ equals the area of $\Delta_{0}$. This allows us to express the radius of the circle $l$ (cf. Thm. 1) in terms of $\Delta_{0}$ 's side lengths:

\section{Corollary 1 The radius of $l$ equals}

$$
R_{1}=\frac{a b c}{a^{2}+b^{2}+c^{2}} \text {. }
$$

Proof. Lengths are scaled with the factor $\lambda$ when applying the similarity $\Delta_{0} \rightarrow \Delta_{1}$. Thus, the circumradius $R_{0}$ changes to $R_{1}=R_{0} \lambda$ with $\lambda$ given in (8). According to the Law of sines, $R_{0}=\frac{a b c}{4 F}$, and thus, $R_{1}=a b c \sigma^{-1}$.

The fact that the sequence of triangles $\Delta_{0}, \Delta_{1}, \Delta_{2}, \ldots$ (as well as the sequence of all $\nabla_{i}$ ) consists of scaled versions of the initial triangle $\Delta_{0}$ together with the fact that the scaling factor depends on the side lengths of each triangle in the same way (cf. Eq. (8)) makes the traces of $\Delta_{0}$ 's vertices a special polygon. For example, the segment $A_{1} A_{2}$ is orthogonal to $A_{0} A_{1}$ and $\overline{A_{1} A_{2}}=\lambda \cdot \overline{A_{0} A_{1}}$. This holds true for any pair $\left(A_{i} A_{i+1}, A_{i+1} A_{i+2}\right)$ of subsequent segments. Thereby, a sequence of discrete equiform motions (consisting of a quarter turn and a constant scaling) moves the polygon $A_{0} A_{1} A_{2} \ldots$ into itself and we can say that $A_{0} A_{1} A_{2} \ldots$ is invariant under the sequence of discrete equiform motions. In the smoth case, the trace of a point undergoing one-parameter equiform motion with constant parameter is a logarithmic spiral. Therefore, we can call $A_{0} A_{1} A_{2} \ldots$ a discrete logarithmic spiral. Fig. 9 shows the six discrete logarithmic spirals traced by the three vertices of $\Delta_{0}$ undergoing the two independent sequences of discrete equiform motions.

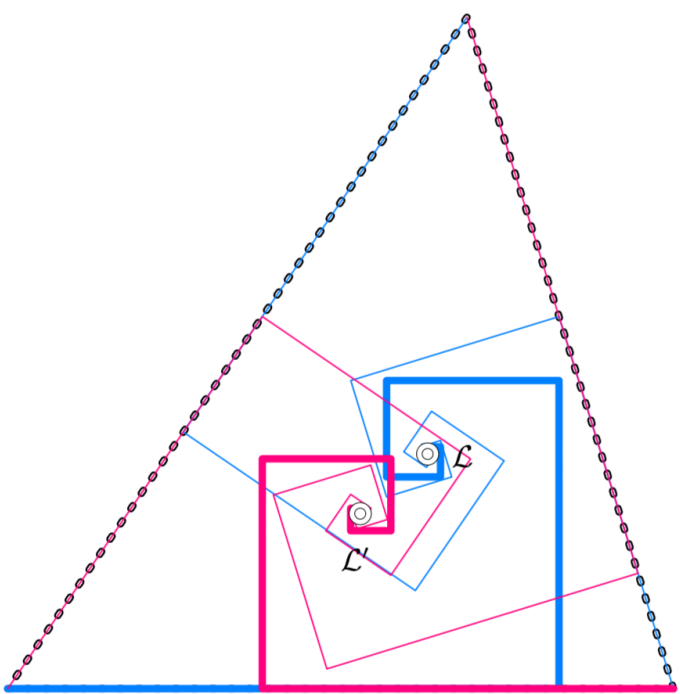

Figure 9: The six discrete logarithmic spirals orbiting the limit points $\mathcal{L}$ and $\mathcal{L}^{\prime}$.

Now, we are able to state and prove:

Theorem 3 The limit position $\mathcal{L}$ of all points $A_{i}, B_{i}$, or $C_{i}$ is the first Brocard point of $\Delta_{0}$ with homogeneous trilinear coordinates

$\mathcal{L}=\left(a c^{2}: b a^{2}: c b^{2}\right)$

while the limit $\mathcal{L}^{\prime}$ of all points $K_{i}, L_{i}$, or $M_{i}$ is the second Brocard point of $\Delta_{0}$, i.e., in terms of homogeneous trilinear coordinates

$\mathcal{L}^{\prime}=\left(a b^{2}: b c^{2}: c a^{2}\right)$.

Proof. With the initial choice of the coordinate frame, we can find the limit position

$$
\mathcal{L}=\lim _{i \rightarrow \infty} A_{i} .
$$


The $x$ - and $y$-coordinate $x_{\mathcal{L}}$ and $y_{\mathcal{L}}$ are

$x_{\mathcal{L}}=\overline{A_{0} A_{1}}-\overline{A_{2} A_{3}}+\overline{A_{4} A_{5}}-\overline{A_{6} A_{7}} \pm \ldots$,

$y_{\mathcal{L}}=\overline{A_{1} A_{2}}-\overline{A_{3} A_{4}}+\overline{A_{5} A_{6}}-\overline{A_{7} A_{8}} \pm \ldots$

The similarity $\Delta_{i} \rightarrow \Delta_{i+1}$ changes lengths by scaling them with the factor $\lambda$, i.e.,

$$
\overline{A_{i} A_{i+1}}=\lambda^{k} \cdot \overline{A_{i+k} A_{i+k+1}}
$$

for $i, k \in\{0, \ldots, n\}$. Consequently, the coordinates $x_{\mathcal{L}}$ and $y_{\mathcal{L}}$ from (11) change to

$$
\begin{aligned}
& x_{\mathcal{L}}=\overline{A_{0} A_{1}}\left(1-\lambda^{2}+\lambda^{4} \mp \ldots\right), \\
& y_{\mathcal{L}}=\overline{A_{0} A_{1}} \lambda\left(1-\lambda^{2}+\lambda^{4} \mp \ldots\right) .
\end{aligned}
$$

The length of the segment $\overline{A_{0} A_{1}}$ follows from (1), (7) with (2) and is already given in (4):

$$
\overline{A_{0} A_{1}}=t=2 b^{2} c \sigma^{-1} .
$$

We let

$\tau:=a^{2} b^{2}+b^{2} c^{2}+c^{2} a^{2}$

and with (8), we can infer

$$
\lambda=\sqrt{\frac{2 \tau-a^{4}-b^{4}-c^{4}}{2 \tau+a^{4}+b^{4}+c^{4}}}<1
$$

for any admissible choice of $a, b$, and $c$. Consequently, the infinite alternating sum of even powers of $\lambda$ attains the value

$$
\frac{1}{1+\lambda^{2}}
$$

which gives

$\mathcal{L}=\frac{b^{2} c}{2 \tau}(\sigma, 4 F)$

Note that $\mathcal{L}$ given in (14) is at the same time the limit of $B_{i}$ and $C_{i}$ too.

The $y$-coordinate of $\mathcal{L}$ in (14) equals the distance of $\mathcal{L}$ to the line $\left[A_{0}, B_{0}\right]$. Therefore, it is the third actual trilinear coordinate of $\mathcal{L}$. It is elementary to compute the first and second actual trilinear coordinate of $\mathcal{L}$ and it turns out that they can be obtained by cyclically replacing $a, b, c$ once and twice in $2 b^{2} c F \tau^{-1}$. We observe that both $F$ and $\tau$ are cyclic symmetric in $a, b, c$, and therefore, they do not change. For the sake of simplicity, we aim at homogeneous trilinear coordinates of $\mathcal{L}$ which allows us to cancel cyclic symmetric factors as long as they are common to all coordinate functions. So, we obtain (9).
A comparison of (9) with the trilinear representation of the first Brocard point given in [6] confirms that $\mathcal{L}$ is indeed the first Brocard point.

The calculations do not really differ if we compute the limit position $\lim _{i \rightarrow \infty} K_{i}=\mathcal{L}^{\prime}$. In this case, it is beneficial to start at $B_{0}=(c, 0)$ and determine

$$
\mathcal{L}^{\prime}=\left(\begin{array}{l}
c \\
0
\end{array}\right)+\overline{B_{0} L_{1}}\left(\begin{array}{c}
-1+\lambda^{2}-\lambda^{4} \pm \ldots \\
\lambda\left(1-\lambda^{2}+\lambda^{4} \mp \ldots\right)
\end{array}\right)
$$

where $\overline{B_{0} L_{1}}=2 a^{2} c \sigma^{-1}$ which results in

$\mathcal{L}^{\prime}=\frac{c}{2 \tau}\left(b^{2} c^{2}-a^{4}+\tau, 4 a^{2} F\right)$.

In the same way as above, we end with

$\mathcal{L}^{\prime}=\left(a b^{2}: b c^{2}: a^{2} c\right)$

Remark 2 The two limit positions $\mathcal{L}$ and $\mathcal{L}^{\prime}$ are no triangle centers: Of course, the trilinear representation is apparently cyclic symmetric in the side lengths $a, b, c$ of $\Delta_{0}$, but, they do not satisfy the norming condition: If $f(a, b, c)$ is a center function, then it also has to satisfy $|f(a, b, c)|=|f(a, c, b)|$ in order to make $(f(a, b, c)$ : $f(b, c, a): f(c, a, b))$ a center.

Now, we show

Theorem 4 The point $\mathcal{L}$ is the only (real and proper) common point of the three Thaloids of the segments $A_{0} A_{1}$, $B_{0} B_{1}, C_{0} C_{1}$.

The point $\mathcal{L}^{\prime}$ is the only (real and proper) common point of the three Thaloids of the segments $A_{0} K_{1}, B_{0} L_{1}, C_{0} M_{1}$.

Proof. From the asymptotic point $\mathcal{L}$ of the discrete logarithmic spiral $A_{0} A_{1} A_{2} \ldots$, any segment $A_{i} A_{i+1}$ can be seen at a right angle, since each subsequent segment of the discrete logarithmic spiral corresponds to a quarter turn plus a scaling with the factor $\lambda$. This is also the case for the segments $B_{i} B_{i+1}$ and $C_{i} C_{i+1}$.

For the same reasons, the limit $\mathcal{L}^{\prime}$ of the second sequence is simultaneously located on three Thaloids.

Thm. 4 provides a very elegant and elementary construction of the two Brocard points $\mathcal{L}$ and $\mathcal{L}^{\prime}$. This construction seems not to be mentioned in the literature. 


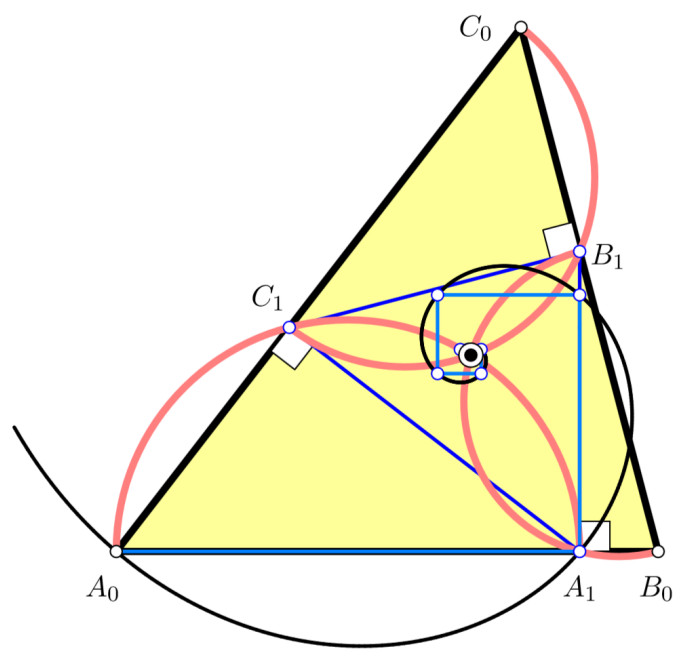

Figure 10: Three Thaloids concur in $\mathcal{L}$.

Fig. 10 shows the three Thaloids through $\mathcal{L}$. Clearly, there are also three Thaloids passing through $\mathcal{L}^{\prime}$.

As a consequence of Thm. 3, the two limits of the triangle tunnels are located on a special self-isotomic pivotal cubic K012 (cf. [1]), better known as the Tucker-Brocard cubic $\mathcal{T}$. Only a few centers from Kimberling's list [5] are known to lie on $\mathcal{T}$ : the symmedian point (also Lemoine point or Grebe point) $X_{6}$, its isotomic conjugate $X_{76}$ (the third Brocard point), and further the two centers $X_{880}$ and $X_{882}$. $\left(X_{882}\right.$ lies on the Brocard axis $\left[\mathcal{L}, \mathcal{L}^{\prime}\right]$.) Fig. 11 shows the tunnel limits (Brocard points) together with the TuckerBrocard cubic $\mathcal{T}$ and the centers $X_{6}, X_{76}, X_{880}, X_{882}$.

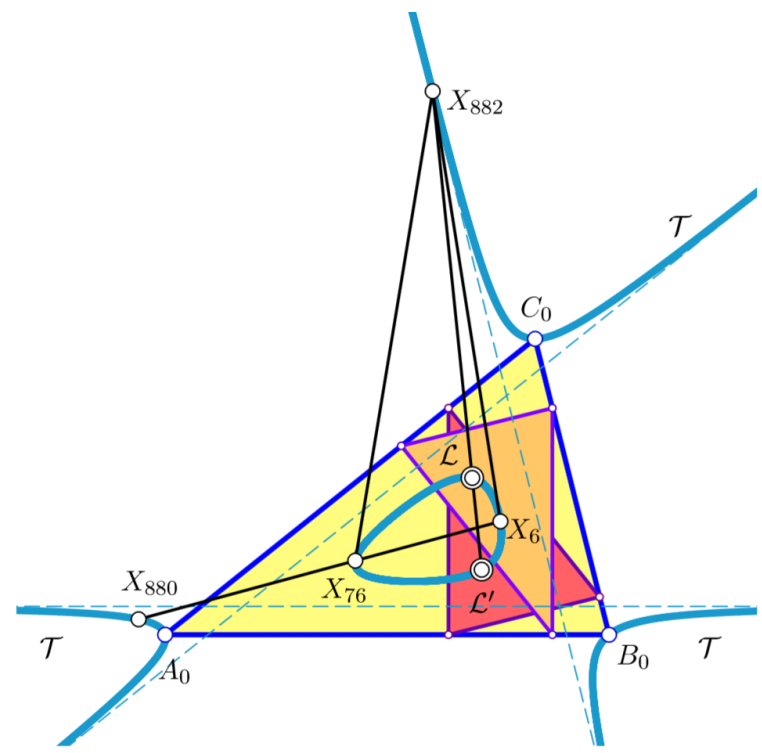

Figure 11: The Tucker-Brocard cubic contains the first and second Brocard point $\mathcal{L}$ and $\mathcal{L}^{\prime}$, besides $X_{6}, X_{76}$, $X_{880}$, and $X_{882}$.

\section{Conclusion and open problems}

The semi-orthogonal paths in quadrilaterals will, in general, not be similar to the initial quadrilateral, for there exists no equiform transformation that maps two quadrilaterals onto each other even when they agree in their interior angles. Nevertheless, the iteration of the computation of semi-orthogonal paths in a quadrilateral produces sequences of shrinking and nested quadrilaterals which preserve their interior angles. Fig. 12 shows an example. In fact, there exist up to six such sequences in a generic quadrilateral. Apparently, there exists an attractor for the six different semi-orthogonal paths in a quadrilateral. It would be interesting to find a tool to compute the limits, if they exist. Shape functions for quadrilaterals as used for triangles in [3] or for generic $n$-gons defined in [10] could help.

Only the case of cyclic quadrilaterals seems hopeful. Since the measures of interior angles are preserved during the transition from one quadrilateral to the next in the sequence of semi-orthogonal paths, so is there sum or difference. In a cyclic quadrilateral opposite interior angels sum up to $\pi$, i.e., $\Varangle A B C+\Varangle C D A=\pi$ and $\Varangle B C D+\Varangle D A B=\pi$ and so do the sums of the opposite (interior) angles in their semiorthogonal paths, see Fig. 13. Hence, the semi-orthogonal path of a cyclic quadrilateral is again a cyclic quadrilateral. Numerical experiments show that each $4 i$-th semiorthogonal path of a cyclic quadrilateral is similar and perspective to the initial cyclic quadrilateral $(i \in \mathbb{N})$. The perspector is common to all pairs of quads, cf. Fig. 14.

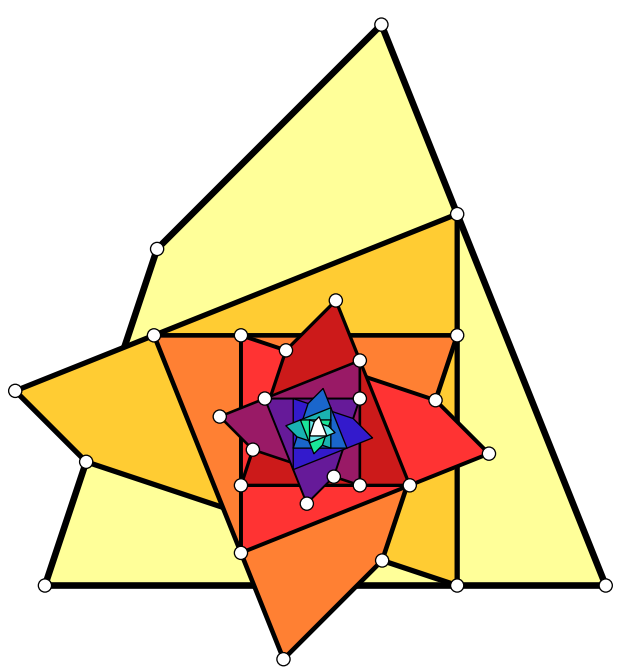

Figure 12: A sequence of shrinking and nested closed semiorthogonal paths in a quadrilateral. It is obvious that interior angles remain unchanged. 


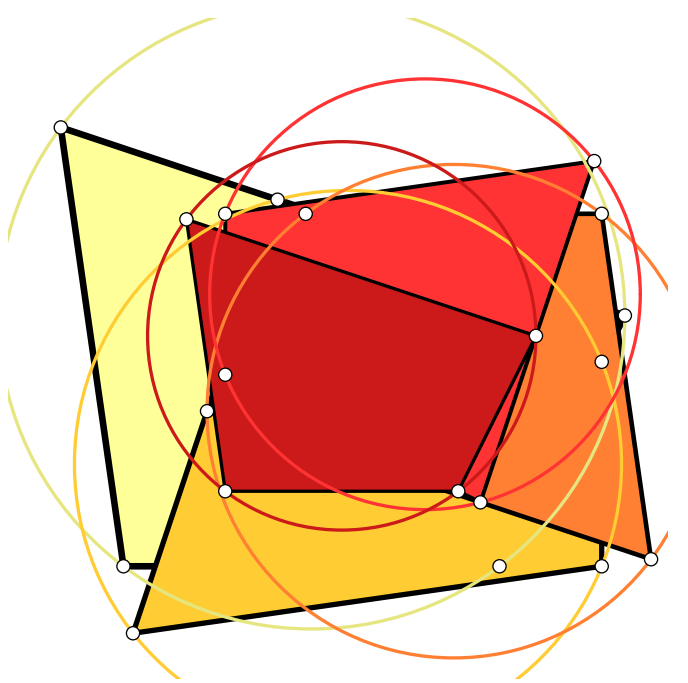

Figure 13: The first four semi-orthogonal paths of a cyclic quadrilateral are similar and perspective to the initial quadrilateral.

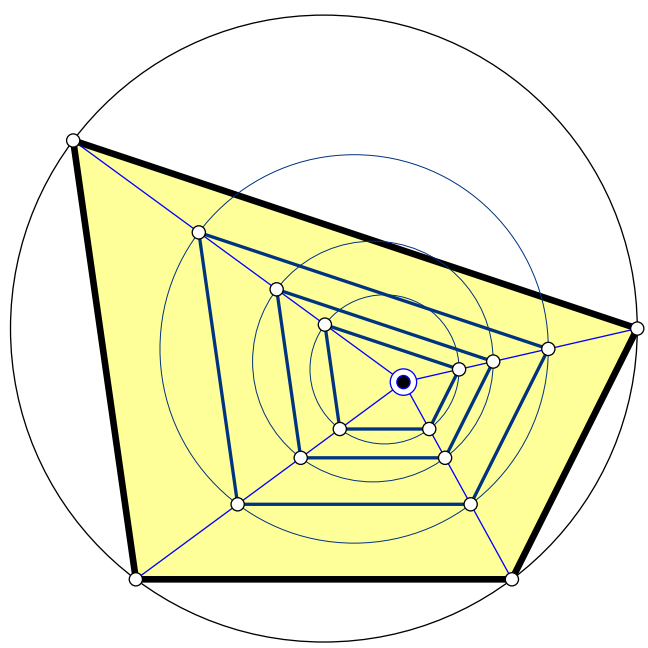

Figure 14: The $4^{\text {th }}, 8^{\text {th }}$, and $12^{\text {th }}$ semi-orthogonal path of a cyclic quadrilateral are similar and perspective to the initial quadrilateral. The perspector could be see as the limit of the sequence of semiorthogonal paths.

\section{References}

[1] B. GIBERT, Tucker-Brocard cubic, Available at: http://bernard.gibert.pagesperso-orange. $\mathrm{fr} /$ Exemples/k012.html

[2] G. Glaeser, H. Stachel, B. Odehnal, The Universe of Concis, From the ancient Greeks to $21^{\text {st }}$ century developments, Springer-Spektrum, SpringerVerlag, Heidelberg, 2016.

[3] M. HajJA, On nested sequences of triangles, Result. Math. 54(3) (2009), 289-299.

[4] C. Kimberling, Triangle Centers and Central Triangles, (Congressus Numerantium Vol. 129), Utilitas Mathematica Publishing, Winnipeg, 1998.

[5] C. Kimberling, Encyclopedia of Triangle Centers, Available at: http://faculty.evansville.edu/ ck6/encyclopedia

[6] C. Kimberling, Bicentric Pairs of Points and Related Triangle Centers, Forum Geom. 3 (2003), 3547.

[7] B. OdehnAL, Generalized Gergonne and Nagel Points, Beitr. Alg. Geom. 51(2) (2010), 477-491.

[8] N. Le, N.J. Wildberger, Universal Affine Triangle Geometry and Four-fold Incenter Symmetry, KoG 16 (2012), 63-80.

[9] N. LE, Four-Fold Symmetry in Universal Triangle Geometry, $\mathrm{PhD}$ thesis, University of New South Wales, Australia, 2015.

[10] E.L. WAChSPRESS, A rational basis for function approximation. Lecture Notes in Mathematics. Vol. 228, Springer, New York, 1971.

\section{Boris Odehnal}

orcid.org/0000-0002-7265-5132

e-mail: boris.odehnal@uni-ak.ac.at

University of Applied Arts Vienna

Oskar-Kokoschka-Platz 2, A-1100 Vienna, Austria 